OPEN Microsecond dynamics of an unfolded

SUBJECT AREAS:

SINGLE-MOLECULE

BIOPHYSICS

PROTEIN FOLDING

CONFOCAL MICROSCOPY

FLUORESCENCE IMAGING

Received

3 December 2012

Accepted

20 June 2013

Published

5 July 2013

Correspondence and requests for materials should be addressed to S.T. (st@tagen.tohoku. ac.jp) \section{protein by a line confocal tracking of single molecule fluorescence}

\author{
Hiroyuki Oikawa ${ }^{1,2}$, Yuta Suzuki 1,3, Masataka Saito 1,3, Kiyoto Kamagata ${ }^{1,2,3}$, Munehito Arai ${ }^{4,5}$ \\ \& Satoshi Takahashi, ${ }^{1,3}$
}

'Institute of Multidisciplinary Research for Advanced Materials, Tohoku University, 2-1-1 Katahira, Aoba, Sendai, Miyagi 9808577, Japan, ${ }^{2}$ Core Research for Evolutional Science and Technology, Japan Science and Technology Agency, 4-1-8 Honcho, Kawaguchi, Saitama 332-0012, Japan, ${ }^{3}$ Department of Chemistry, Graduate School of Science, Tohoku University, Aramaki, Aoba, Sendai, Miyagi 980-8578, Japan, ${ }^{4}$ PRESTO, Japan Science and Technology Agency, 4-1-8 Honcho, Kawaguchi, Saitama 332-0012, Japan, ${ }^{5}$ Department of Life Sciences, Graduate School of Arts and Sciences, The University of Tokyo, 3-8-1 Komaba, Meguro, Tokyo 153-8902, Japan.

We present a new method for high speed tracking of fluorescence time series from single proteins. The method uses a fast sample flow and a modified confocal microscopy, line confocal microscopy, and achieves the time resolution of less than $20 \mu \mathrm{s}$. The obtained time series from the B domain of protein A labeled with donor and acceptor fluorophores suggest conformational heterogeneity and dynamic fluctuations in the unfolded state.

ingle molecule fluorescence spectroscopy (SMFS) has evolved as a powerful strategy for the structural and dynamical characterization of biological macromolecules ${ }^{1-3}$. In typical SMFS experiments, fluorescence photons from both donor and acceptor fluorophores labeled to a sample are detected simultaneously, and are analyzed based on the Förster resonance energy transfer (FRET) mechanism to obtain the inter-fluorophore distance. The power of SMFS is typically exemplified in the characterization of the unfolded proteins at the single molecule level ${ }^{4-7}$. The structural and dynamical properties of the unfolded proteins can be routinely obtained by using SMFS. However, the time resolution of SMFS in obtaining continuous time series is usually limited to a few milliseconds. Furthermore, the noise width of the FRET efficiency measurements is significant. Accordingly, conformations having lifetimes shorter than the time resolution or those having similar FRET efficiencies cannot be resolved. Here, we developed a new strategy for SMFS data acquisition that significantly improves the time resolution and the noise width by increasing the number of photons detected in a unit time (photon count rate).

The photon count rate in SMFS is mainly determined by the photodynamics of fluorophores. In a confocal method, the common data acquisition method of SMFS, a single protein in a small focal volume of an objective is excited and the fluorescence photons are collected by the same objective and detected by avalanche photodiodes. The photon count rate in this method saturates easily at a relatively low excitation power because of the blinking phenomenon caused by the interruption of the photocycle between the ground singlet state $\left(\mathrm{S}_{0}\right)$ and the excited singlet state $\left(\mathrm{S}_{1}\right)$ due to the formation of the triplet state $\left(\mathrm{T}_{1}\right)$ and its subsequent conversion to dark states with relatively long lifetime. In addition, the bleaching reaction mainly between the fluorophore and singlet oxygen $\left({ }^{1} \mathrm{O}_{2}\right)$, generated by the collision of $\mathrm{T}_{1}$ with triplet oxygen $\left({ }^{3} \mathrm{O}_{2}\right)$, occurs at stronger excitations. A removal of dissolved ${ }^{3} \mathrm{O}_{2}$ from the sample solution is used to reduce the fluorophore bleaching. However, since ${ }^{3} \mathrm{O}_{2}$ acts as a strong quencher for $\mathrm{T}_{1}$, the removal frequently leads to an increase of the blinking. Thus, the photon count rate in the confocal method of SMFS is usually limited to $\sim 30$ photons $/ \mathrm{ms}$. Accordingly, it is difficult to resolve events occurring within a diffusive residence time (several milliseconds) of a molecule in a focal volume of an objective.

Several possibilities have been proposed to increase the photon count rate in SMFS by minimizing the effects of the photodynamical events. Using trolox and dissolved ${ }^{3} \mathrm{O}_{2}$ to minimize the $\mathrm{T}_{1}$ formation and cysteamine to scavenge ${ }^{1} \mathrm{O}_{2}$, Campos et al. achieved the photon count rate of $\sim 800$ photons $/ \mathrm{ms}^{8}$. However, the additives quenched fluorescence emission, and their photoprotection effects were dependent on fluorophores ${ }^{8}$. Another possibility to increase the photon count rate is the use of fast sample flow without using additives ${ }^{9}$. In the ensemble measurements based on the confocal system under the fast sample flow, in which $\sim 3$ fresh molecules were 
continuously supplied to the focal volume, Horrocks et al. reported the photon count rate of more than $\sim 1600$ photons/ms per molecule $^{9}$. However, the flow system cannot be easily combined with the confocal detection of SMFS, since the residence time of a molecule in the focal volume of objectives becomes extremely short under the flow, making the continuous observation of single molecules difficult. In this investigation, we introduced a new data acquisition strategy in SMFS, a line confocal microscopy, to achieve a higher photon count rate and a longer observation time at the same time.

\section{Results}

We developed a new approach to simultaneously achieve a higher photon count rate and a longer observation time in SMFS. First, to increase the photon count rate by suppressing the photodynamics without any additives except the dissolved ${ }^{3} \mathrm{O}_{2}$, we used a fast sample flow based on microfluidic cell. Second, to extend the observation time of single molecules under a fast flow, we introduced a line confocal system, which can expand the focal volume to the direction of the sample flow (Fig. 1a). Here, a sample solution flows in a microfluidic chip with a channel of $20-\mu \mathrm{m}$ width and $5-\mu \mathrm{m}$ depth. An objective with a numeric aperture of 0.75 is used for focusing an excitation laser to the flow channel and collecting fluorescence photons from samples; however, the excitation laser is expanded in the flow direction by a cylindrical lens. The collected fluorescence photons are passed through a slit, separated into the donor and acceptor photons, and imaged on an intensified two-dimensional detector. The images of the fluorescence traces are read out after the flow, which are converted to time series of FRET efficiency.

To examine the feasibility of the system, we investigated the equilibrium unfolding transition of the $\mathrm{B}$ domain of protein $\mathrm{A}$ (BdpA), which is a three-helix bundle composed of 60 residues ${ }^{10,11}$. We introduced two cysteines at residues 22 and 55, which were labeled by Alexa488 and Alexa633 (Fig. 1b) ${ }^{12}$. The labeled protein showed a two-state unfolding transition by guanidium chloride $(\mathrm{GdmCl})$ (Supplementary Fig. S1) ${ }^{10-12}$. The single molecule fluorescence images of the native protein at $1 \mathrm{M} \mathrm{GdmCl}$ observed at the flow speed of $300 \mathrm{nl} / \mathrm{min}$ were converted to the time series of fluorescence intensity (Fig. 1c). Fluorescence intensity distribution of one trace is highest around the center of the channel and lower at the two ends, reflecting the spatial differences in the efficiency of laser excitations and of collecting photons. In addition, there exist short-term fluctuations of the fluorescence intensities for the donor and acceptor, which are likely attributed to the sample diffusion. These intensity fluctuations are cancelled by calculating the FRET efficiency (Fig. 1c,d). The photon count rate for the donor channel is typically 2000 photons/ms. The average FRET efficiency for each trace does not depend on the fluorescence intensity, supporting that the photodynamics occurring at stronger excitations do not affect the observed data (Supplementary Fig. S2). Based on the passage time of a molecule in one pixel $(\sim 60 \mu \mathrm{s}$, Supplementary Text) and the blurring of the image (less than 2 pixels), the time resolution of these traces is less than $120 \mu \mathrm{s}$. Thus, our line confocal system achieves the photon count rate of more than 2000 photons/ms for more than five milliseconds.

The FRET efficiency distribution of BdpA at $1 \mathrm{M} \mathrm{GdmCl}$ shows a peak for the native protein (Fig. 2a). The estimated noise width determined by the photon shot noise and intensifier noise (dashed line) coincides with the observed distribution. In addition, the standard deviation for each trace divided by the estimated noise width (i.e., the relative fluctuation) is distributed to the values slightly larger than one, suggesting that the fluctuation within each trace is explained by the noise (Fig. 2b, Supplementary Text). The data in the presence of $1.5 \mathrm{M} \mathrm{GdmCl}$ have two distributions. The major distribution corresponds to the native state and is shifted to a lower efficiency as observed in the ensemble measurements (Fig. 2a and Supplementary Fig. S1); however, the relative fluctuation is similarly distributed to the values slightly larger than one (Fig. 2b). Thus, the data suggest the conformational homogeneity and the absence of the detectable dynamics for the native state of BdpA.

By contrast, the data obtained for the unfolded BdpA suggest structural heterogeneity and dynamic fluctuations. The FRET distributions at $\geq 2 \mathrm{M} \mathrm{GdmCl}$ are significantly broader than the noise width (Fig. 2a), suggesting structural heterogeneity in the unfolded $\mathrm{BdpA}$. In addition, the relative fluctuations are distinctly larger than the values obtained for the native state (Fig. 2b), suggesting that the fluctuations in the FRET efficiency are caused by protein dynamics.
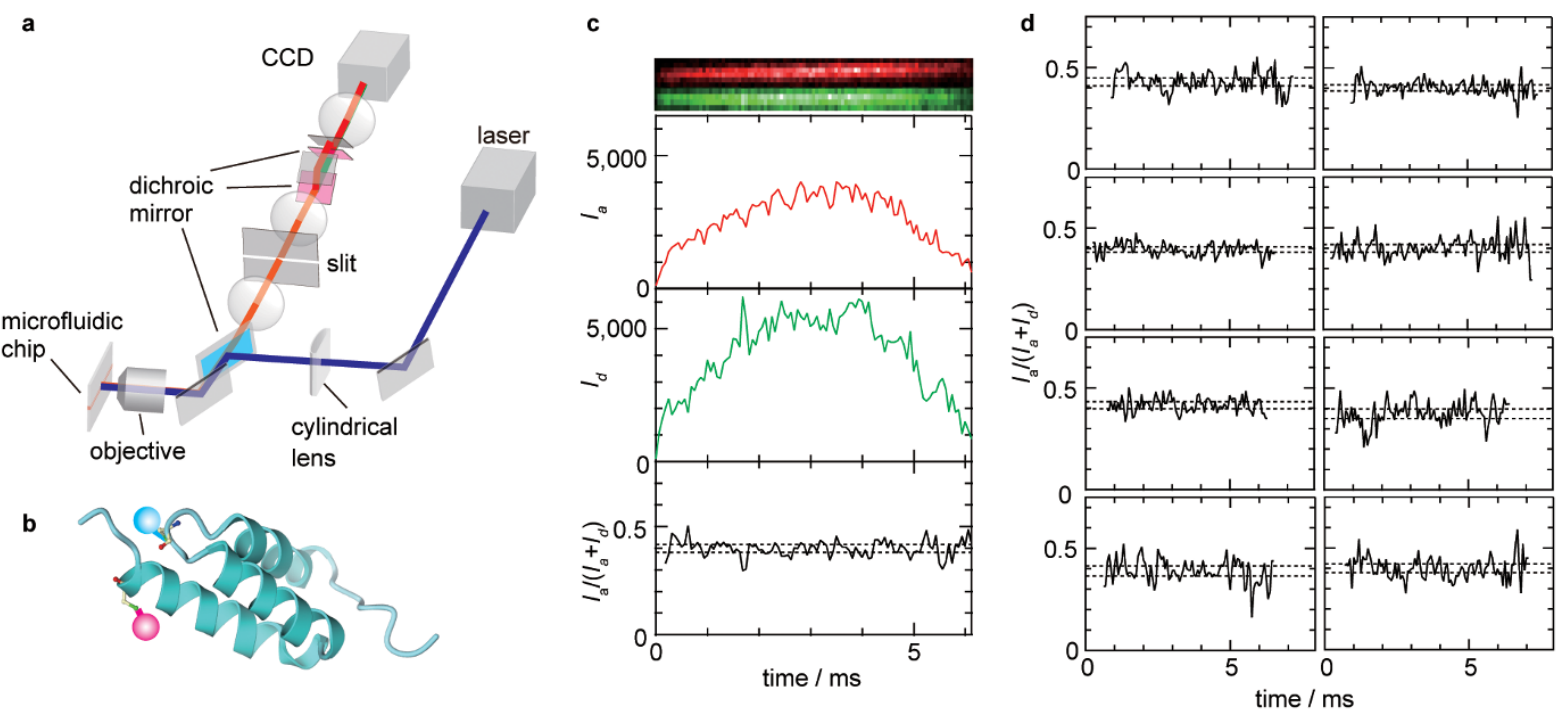

Figure $1 \mid$ (a) The optical set up of the line confocal system for SMFS. See text for details. (b) The native structure of BdpA. The two labeling sites, residues 22 and 55, are indicated by colored spheres. (c) Examples of the single molecule data obtained by the system. (Upper) Raw fluorescence images of the flowing BdpA at the donor (green) and acceptor (red) channels. The image was pseudo colored. (Middle) The red and green traces show the raw fluorescence intensity time series for the acceptor and donor photons, respectively, calculated from the upper image. (Lower) The black trace shows the apparent FRET efficiency time series calculated by dividing acceptor fluorescence intensity by the sum of the donor and acceptor fluorescence intensities. A dotted line represents the noise width determined by the photon shot noise and the intensifier noise. (d) The FRET efficiency time series obtained for the labeled BdpA at $1 \mathrm{M} \mathrm{GdmCl}$. Dotted lines in each panel represent the noise width of each trace. 

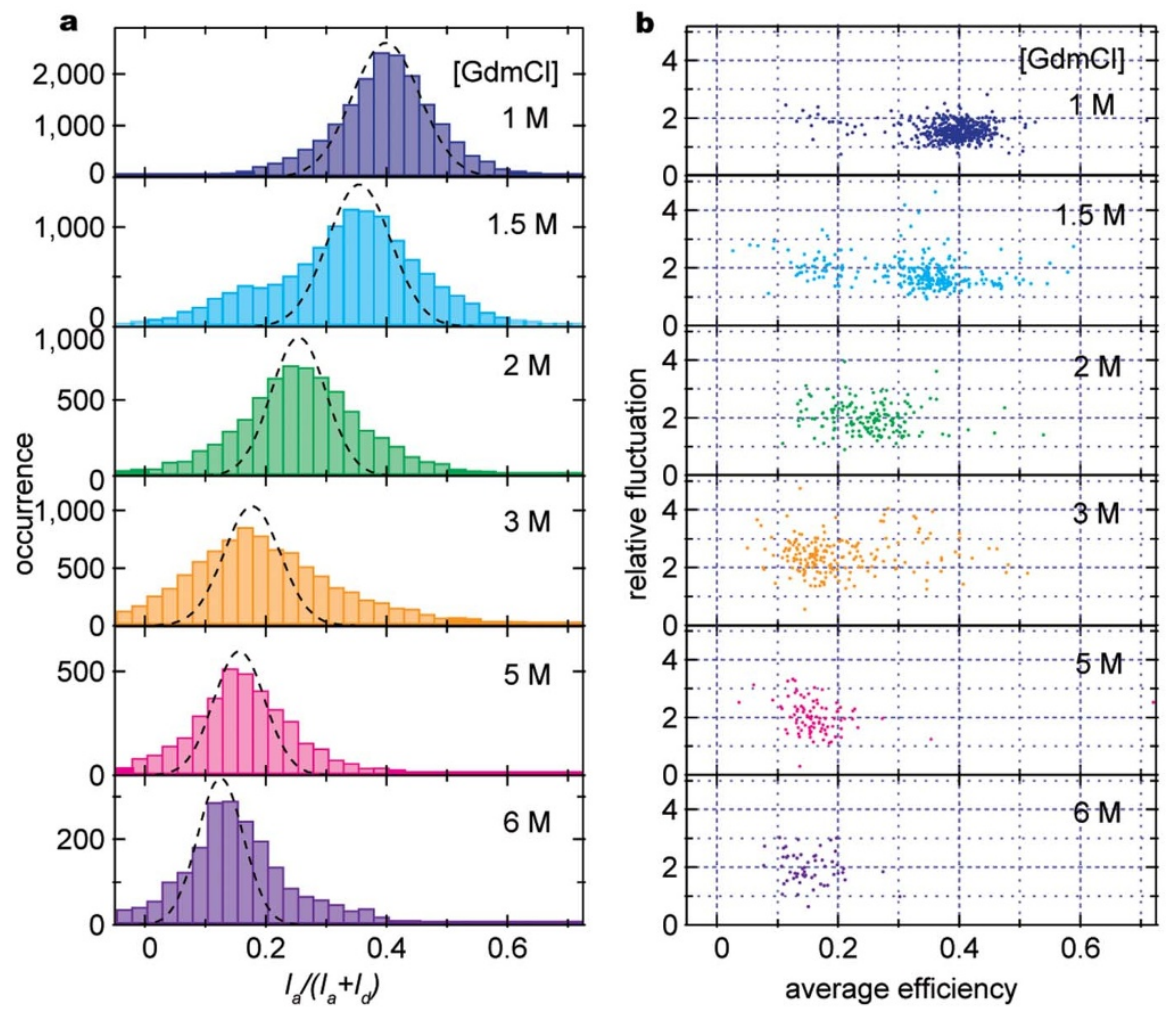

Figure $2 \mid$ (a) The FRET efficiency distributions for the doubly-labeled BdpA obtained at various GdmCl concentrations. Single molecule FRET time series was obtained at the time resolution of less than $120 \mu \mathrm{s}$, from which the FRET distributions were calculated. Dashed lines show the noise width determined by the photon shot noise and the intensifier noise. (b) The scattering plot of the relative fluctuation (ordinate), which is the standard deviation for each trace divided by the estimated noise width, versus the average FRET efficiency (abscissa).

In a time-domain view, some time traces show nearly constant FRET efficiencies; however, the FRET values differ from trace to trace (Fig. 3a and Supplementary Fig. S3), again suggesting the structural heterogeneity. Strikingly, some traces show jumps among slightly different FRET efficiencies, suggesting dynamic fluctuations of an unfolded protein. We further measured the unfolded BdpA at the fastest flow speed of $1500 \mathrm{nl} / \mathrm{min}$ corresponding to the time resolution of less than $20 \mu$ s (Fig. 3b). While there exist traces showing jumps in the FRET efficiency, many traces demonstrate rather constant FRET values over the observation time of several hundreds of
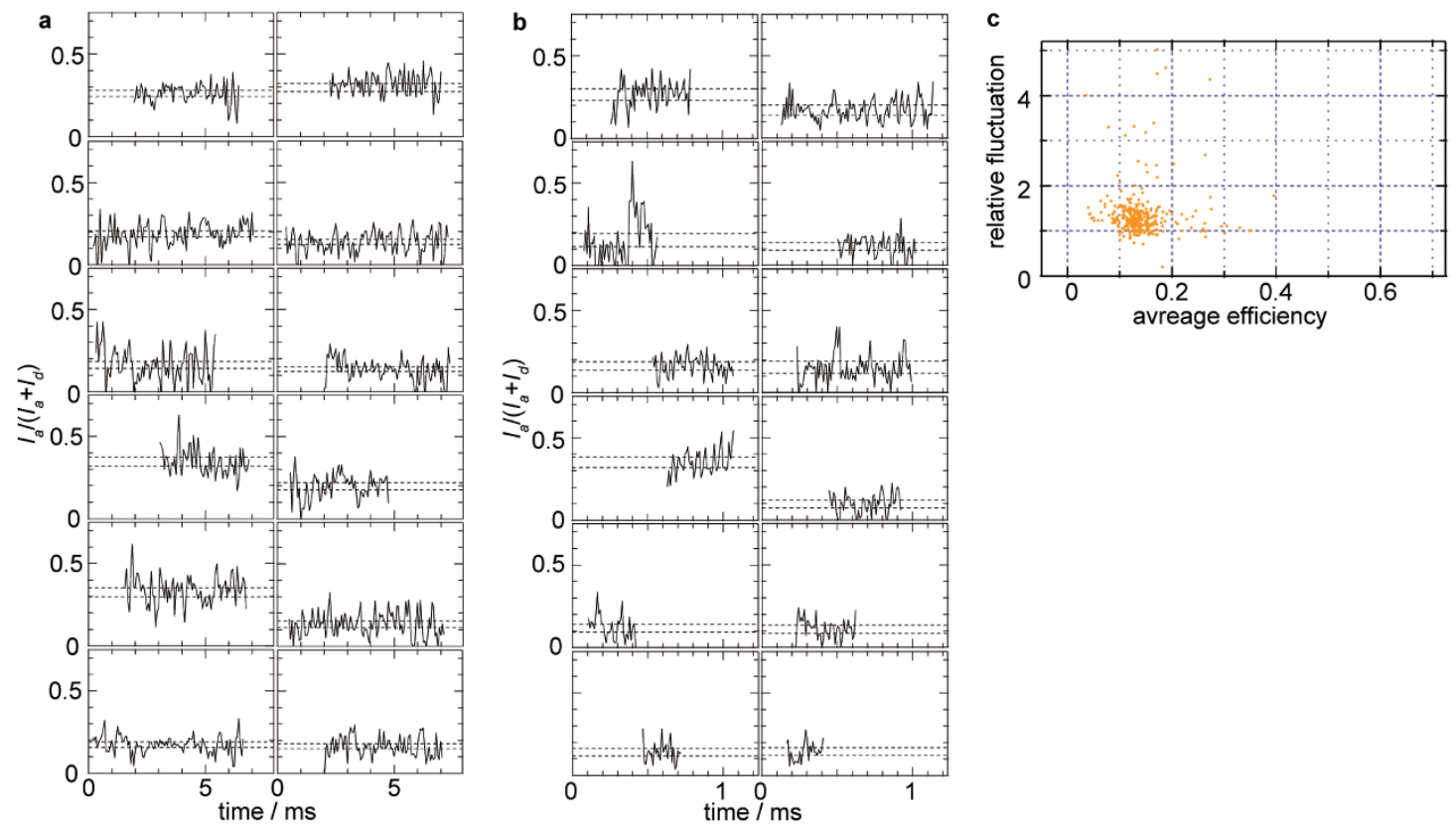

Figure 3 Single molecule FRET results of the doubly-labeled BdpA at 3 M GdmCl. (a,b) The FRET efficiency time series obtained at the time resolution of less than $120 \mu \mathrm{s}(\mathrm{a})$ and $20 \mu \mathrm{s}$ (b). Dotted lines represent the noise width of each trace. (c) The scattering plot of the relative fluctuation (ordinate) and the average FRET efficiency (abscissa) obtained at the time resolution of less than $20 \mu$ s. 
microseconds. The relative-fluctuation plot also supports that the fluctuation is less apparent (Fig. 3c). Therefore, we suggest that the unfolded BdpA shows the significant conformational heterogeneity and the dynamic fluctuations occurring in the time domain longer than several hundreds of microseconds.

\section{Discussion}

We demonstrated that the single-molecule observation system based on the fast sample flow in the presence of ${ }^{3} \mathrm{O}_{2}$ could achieve the photon count rate of more than 2000 photons/ms, which is significantly larger than that usually observed by the conventional method without flow ( $\sim 30$ photons $/ \mathrm{ms})$. The presence of ${ }^{3} \mathrm{O}_{2}$ is important for the efficient quenching of the $\mathrm{T}_{1}$ state of fluorophore (Supplementary Text). To understand the photodynamics in the presence and absence of flow, we examined the dependency of the ensemble fluorescence intensity on the flow rate. We observed that the intensity increases only weakly upon a large increase of the flow rate from $50 \mathrm{nl} / \mathrm{min}$ to $1500 \mathrm{nl} / \mathrm{min}$ (Supplementary Fig. S4a). However, we observed that the ensemble fluorescence intensity becomes weak upon the stopping of sample flow. These observations are in accordance with the previous report ${ }^{9}$, and suggest that the sample flow suppresses the bleaching and blinking of a large fraction of fluorophores by reducing the residence time in the excitation flux. In contrast, a faster flow speed is necessary to suppress the bleaching and blinking at the single molecule level. It was shown that the frequency of observing single molecule traces with higher photon count rate becomes smaller at the slow flow rate (Supplementary Fig. S4b). It can be suggested that samples flowing in the center of the channel, which are excited strongly and are observed as bright traces in the single molecule measurements, are selectively bleached or trapped to a dark state before entering into the observation area at the slow flow rate. We propose that the fast sample flow in the presence of ${ }^{3} \mathrm{O}_{2}$ suppresses the bleaching and blinking of fluorophores under strong excitation, and increases the frequency of observing bright fluorescence traces.

An advantage of using the line confocal microscopy over the point confocal microscopy is longer residence time of a sample in the observation area even in the presence of fast flow. The residence time of a flowing sample in the point confocal microscopy easily becomes several tens of microseconds, making the time series detection of dynamical events difficult ${ }^{9}$. We note that a diffusive movement of a molecule in the laminar flow inside of the channel does not introduce significant artifact on the fluorescence intensity and flow speed (Supporting Text). Assuming a reasonable diffusion constant for $\mathrm{BdpA}\left(1.0 \times 10^{-6} \mathrm{~cm}^{2} / \mathrm{s}\right)$, the mean displacement of BdpA during the observation time ( $5 \mathrm{~ms}$ ) is estimated as $1 \mu \mathrm{m}$, which is within the dimensions of the excitation volume (several micrometers of width and depth). Thus, the fluctuation of donor or acceptor intensities caused by diffusion is not significant, and can be canceled by calculating the FRET efficiency. For example, $\sim 15 \%$ of the short-term intensity fluctuation of the donor trace shown in Fig. 1c was suppressed in the FRET efficiency trace. The flow in the observation channel is a laminar flow with parabolic velocity profile. Assuming that a molecule, initially located in the middle of the cross section of the flow channel (5 $\mu \mathrm{m}$ depth and $20 \mu \mathrm{m}$ width), diffuses to the vertical direction (the $5 \mu \mathrm{m}$ direction) for $1 \mu \mathrm{m}$, the flow speed of the molecule becomes $\sim 16 \%$ slower than the initial speed, which is within the standard deviation of the measured flow speed $(73 \pm$ $18 \mu \mathrm{m} / \mathrm{ms}$, Supporting Text). We conclude that the line confocal microscopy is a practical method of SMFS for the detection of fluorescence time series under the fast sample flow.

$\mathrm{BdpA}$ is one of the typical two-state proteins, and no intermediate conformations with significant populations were reported in the previous ensemble and single molecule investigations ${ }^{10-12}$. The substates suggested in this study may correspond to unresolved conformations in the unfolded $\mathrm{BdpA}$ or might be intermediate conformations caused by reduced cooperativity in the unfolding transition of the doubly-labeled sample. Further investigations are underway using different labeling sites to reveal implication of the substates in the folding of BdpA. In contrast to the start-stop experiment that achieved nanosecond time resolution in the autocorrelation analysis ${ }^{13}$, our method is designed to directly follow a single molecule time series as an experimental counterpart of molecular dynamics calculations ${ }^{14}$. With the improved time resolution and noise width, our method offers a new approach for the time-series investigation of protein folding and other biological phenomena.

\section{Methods}

Optical system. The excitation light was obtained from a diode-pumped solid-state laser (SDL-473-LN-150T, Shanghai Dream Lasers Technology, Shanghai, China), whose wavelength and power were $473 \mathrm{~nm}$ and $150 \mathrm{~mW}$, respectively. The laser output was collimated by a set of two lenses $(f=30 \mathrm{~mm}$ and $80 \mathrm{~mm})$ to obtain a semiGaussian beam with a diameter of $4 \mathrm{~mm}$, passed through a cylindrical lens $(f=$ $70 \mathrm{~mm}$ ), and introduced to an objective (N.A. $=0.75$, CFI S Fluor $20 \times$, Nikon, Tokyo, Japan) using a dichroic mirror (Di01-R488-25 $\times 36$, Semrock, New York, USA). At the focal plane of the objective, the beam forms an elliptic spot whose lengths of major and minor axes were $\sim 0.4 \mathrm{~mm}$ and $\sim 2 \mu \mathrm{m}$, respectively. The excitation irradiance was $75 \sim 150 \mathrm{~kW} / \mathrm{cm}^{2}$ assuming homogeneous excitation at the focusing area. While the irradiance at the center of the area should be stronger than the average, we estimate that the irradiance did not exceed $\sim 500 \mathrm{~kW} / \mathrm{cm}^{2}$. The fluorescence emitted from the sample molecules was collected by the objective, passed through the dichroic mirror, and was spatially filtered by a slit whose width was set at $50 \mu \mathrm{m}$. The expansion of the image at the slit was 5 times. The fluorescence was collimated, separated into the components from the donor and acceptor dyes by using dichroic mirrors (NT49-471, Edmund Optics, New Jersey, USA) and bandpass filters (FF01-536/40-25 and FF01-655/40-25, Semrock), and focused on the imaging detector. The total magnification of the image at the detector was 5 times. The optical system was designed and constructed by Optonica (Kyoto, Japan). The fluorescence was detected by using electron-bombarded CCD combined with image amplifier (C9400-01S, Hamamatsu Photonics, Hamamatsu, Japan). The total gain was set to 120. The fluorescence images from the donor and acceptor dyes were simultaneously detected in one frame by adjusting the separation of the two images appropriately.

Flow system. The sample solution was introduced to a custom-designed microfluidic chip (Institute of Microchemical Technology, Kanagawa, Japan). The observation channel was grooved using a wet etching on a fused silica plate with a thickness of $0.7 \mathrm{~mm}$. The width, depth and length of the channel were $5 \mu \mathrm{m}, 20 \mu \mathrm{m}$ and $2 \mathrm{~mm}$, respectively. The observation channel was connected to the entrance and exit channels with $200-\mu \mathrm{m}$ depth and $400-\mu \mathrm{m}$ width constructed by a mechanical machining. A quartz cover plate with a thickness of $0.2 \mathrm{~mm}$ was covered over the grooves. The single molecule observation was conducted from the cover-plate side by aligning the rectangle excitation area with the observation channel. Before the flow measurements, the sample solution was introduced to a teflon tubing with an inner diameter of $0.25 \mathrm{~mm}$ connected to the microfluidic chip using a teflon holder. A lowvolume, high-pressure chromatography pump (DiNa-SS, KYA Technologies, Tokyo, Japan) was used to push the sample solution at the volumetric flow rate of either 300 and $1500 \mathrm{nl} / \mathrm{min}$. For the stabilization of the sample flow, we waited for at least five minutes after adjusting the setting of the flow pump.

Time resolution. The exposure time of the detector was set at 5.9 and $1.2 \mathrm{~ms}$, respectively, for the measurements at the sample volumetric flow rate of 300 and $1500 \mathrm{nl} / \mathrm{min}$. We estimated that the average line speed of the flowing molecules was 73 (s.d. $=18) \mu \mathrm{m} / \mathrm{ms}$ at the flow rate of $300 \mathrm{nl} / \mathrm{min}$ based on the distances traveled by 19 molecules in the dead time of the camera (Supplementary Text). The average line speed was 390 (s.d. $=48) \mu \mathrm{m} / \mathrm{ms}$ at the flow rate of $1500 \mathrm{nl} / \mathrm{min}$ based on 22 trajectories observed under the modulation of excitation light by an optical chopper (Supplementary Text). The corresponding residence times of a molecule at one pixel were 59 (17) and $10(1.4) \mu \mathrm{s} /$ pixel at the flow rates of 300 and $1500 \mathrm{nl} / \mathrm{ms}$, respectively. Since the current optical system could image a fixed molecule as a spot with a width of less than 2 pixels, the time resolution of the fluorescence time series were less than 120 and $20 \mu$ s at the flow rate of 300 and $1500 \mathrm{nl} / \mathrm{min}$, respectively.

Expression and purification of BdpA. A gene fragment of the triple mutant of BdpA (Y15F/N22C/A55C) with an N-terminal His tag (MGHHHHHHSSG) and a subsequent thrombin cleaving site (LVPRGS) were synthesized using overlap extension PCR with four kinds of oligonucleotides (90-110 bp) purchased from JbioS Ltd. (Saitama, Japan). For constitutive expression of the gene in Escherichia coli, the sigma promoter sequence and the Shine-Dalgarno sequence were attached at the upstream of the BdpA gene as described previously ${ }^{15}$. The amplified gene fragment was digested with BamHI/EcoRI and ligated into the pUC19 plasmid (Takara Bio Inc., Shiga, Japan). The Y15F mutation was introduced to remove intrinsic fluorescence in the previous report ${ }^{12}$. We used the same mutation to compare the results. The E. coli JM109 cells containing the plasmid vector were grown overnight at $37^{\circ} \mathrm{C}$ in the $4 \mathrm{~L}$ of $2 \times$ YT medium containing $60 \mu \mathrm{g} / \mathrm{ml}$ ampicillin, and were harvested by centrifugation. The cell pellet was resuspended in buffer A (20 mM Tris- 
$\mathrm{HCl}(\mathrm{pH} 8.0)$ and $200 \mathrm{mM} \mathrm{NaCl}$ ), and was lysed by French Press. The supernatant fraction after the addition of streptomycin and $40 \%$ ammonium sulfate was collected, and was loaded onto a NiNTA Superflow (Qiagen) gravity column in the presence of $20 \mathrm{mM}$ imidazole. Following extensive washing with buffer A, the column was incubated overnight under gentle shaking at room temperature in the presence of 100 units of thrombin (Nakalai Tesque, Kyoto, Japan). The cleaved BdpA was eluted in the flow-through of the gravity column. The protein was further purified by DEAE ion exchange chromatography (GE healthcare, Buckinghamshire, UK) in the presence of $1 \mathrm{mM}$ dithiothreitol (DTT). The purity and molecular weight of the protein were checked by SDS-PAGE and liquid chromatography mass spectrometry. Protein concentration was determined using the BCA protein assay kit (Pierce). About $150 \mathrm{mg}$ of the protein was obtained from the $4 \mathrm{~L}$ culture. The primary sequence of the BdpA mutant was GS-TADNKFNKEQQNAFFEILHLPCLNEEQRNGFIQSLKDDPSQSANLLAEAKKLNDCQAPKA (60 + 2 residues), in which the mutation sites are underlined.

Protein labeling. The purified sample solution was stored as the ammonium sulfate precipitate at $4{ }^{\circ} \mathrm{C}$. The collected precipitate was dissolved in buffer B $(50 \mathrm{mM}$ Tris$\mathrm{HCl}$ at $\mathrm{pH} 7.4$ and $100 \mathrm{mM} \mathrm{NaCl}$ ) with $1 \mathrm{mM} \mathrm{DTT}$, and dialyzed against the same buffer to remove ammonium sulfate and to reduce the disulfide bonds. The sample buffer was exchanged to buffer B using a PD-10 desalting column (GE Healthcare) to remove DTT. The protein solution was concentrated to $12 \mu \mathrm{M}$ using Amicon Ultra-4 centrifugal filter unit with $3 \mathrm{kDa}$ nominal molecular weight limit (Millipore, Massachusetts, USA). To this solution, a 1.3-fold excess of Alexa Fluor 633 maleimide (Invitrogen, Oregon, USA) was added and incubated at room temperature for one hour, and further at $4^{\circ} \mathrm{C}$ for overnight. The unreacted dye was removed by the PD-10 column, and the protein solution was concentrated to $10 \mu \mathrm{M}$. The solution was purified by HiTrap Q HP (GE Healthcare) anion exchange chromatography, by using a linear gradient of 20 column volumes with an increasing ionic strength up to $1 \mathrm{M}$ $\mathrm{NaCl}$ in a $20 \mathrm{mM}$ Tris- $\mathrm{HCl}(\mathrm{pH}$ 7.4). The colored fractions were examined by a matrix-assisted laser desorption/ionization time-of-flight mass spectroscopy (MALDI-TOF MS), and fractions containing singly-labeled protein were collected. The buffer solution of the singly-labeled protein was exchanged to buffer $\mathrm{B}$. The sample concentration was adjusted at $50 \mu \mathrm{M}$. To this solution, 10 -fold excess of Alexa Fluor 488 maleimide and a 10-fold excess of tris(2-carboxyethyl)-phosphine hydrochloride were added, and incubated at room temperature for one hour and at $4^{\circ} \mathrm{C}$ for overnight. The doubly-labeled protein was purified by HiTrap Q HP anion exchange chromatography, and confirmed by MALDI-TOF MS

Single molecule measurements. The sample for the single molecule measurements were prepared by dissolving the stock solution of the doubly-labeled protein into the observation buffer containing $20-\mathrm{mM}$ Tris- $\mathrm{HCl}(\mathrm{pH} 7.4)$ and various concentrations of $\mathrm{GdmCl}$. The buffer solution was filtered by membrane filter with $0.22-\mu \mathrm{m}$ pores (Millex-GS, Millipore, USA). Typically, $80 \mu \mathrm{l}$ of the sample solution adjusted at concentrations between 0.1 and $1 \mathrm{nM}$ were prepared, and used for the detection of several hundreds of single molecule traces over the observation time of more than four hours. All the single molecule measurements were conducted in a room, in which the temperature was regulated at $21 \sim 23^{\circ} \mathrm{C}$. In the case for the current sample, the single molecule measurements were difficult in the absence of $\mathrm{GdmCl} \mathrm{likely} \mathrm{due} \mathrm{to} \mathrm{the}$ adsorption of sample on the surface of quartz cell (c.f. Supplementary Fig. S1b). We therefore used the solution containing $1-\mathrm{M} \mathrm{GdmCl}$ as the sample for the native state.

Data analysis. Raw data of the fluorescence images of the flow cell containing more than million frames were stored without data processing. Most of the stored images correspond to blank images without flowing molecules. The ratio of the images containing signals from fluorescent molecules over the blank images was roughly one frame among $5000 \sim 10000$ frames. To extract single molecule time series from the observed frames, we conducted two steps of screening. In the first step of screening, we selected candidate frames that may contain signals from single molecules. In the second step of screening, we eliminated regions of the candidate frames where signals are absent.

In the first step of screening, we selected the candidate frames that may contain signals from fluorescent molecules. We first selected the regions of frames corresponding to donor and acceptor channels. Next, the pixel intensities of the flow channel were binned for 6 pixels to the direction perpendicular to the flow direction, and the frames having three adjacent pixels along the flow direction with intensities higher than the twice of the background noise were selected. If the above criterion was satisfied in either the donor or the acceptor images of a frame, the frame was considered as a candidate frame containing single molecule data.

From the candidate frames, we next subtracted background intensity levels. For the candidate frames, we returned to the original two-dimensional images before the binning. We collected 500 frames before and after one candidate frame (totally 1000 frames), averaged them as the averaged background image and subtracted it from the image in the candidate frame. The procedure was repeated for all the candidate frames, and can compensate for possible long-term drifts in the laser intensity, the detector gain and the defocused fluorescence background. In the donor channel, the background level was typically 400 counts/pixel at the exposure time of $6 \mathrm{~ms}$, corresponding to 1.7 photons/ms/pixel. In the acceptor channel, the background level was nearly zero.

The candidate frames thus selected still contain regions where fluorescent molecules were absent. In addition, since the threshold levels used in the first level of screening were set to rather low values, the selected frames contain very weak signals.
We accordingly performed the second level of screening to select single molecule traces having intensities significantly higher than the noise. The fluorescence intensity corresponding to the axis perpendicular to the flow axis was again binned for 6 pixels for both of the donor and acceptor channels. If the data count for the sum of the donor and acceptor channels are higher than 1100 and 400 counts for the more than five adjacent pixels for the measurements conducted at the flow rates of 300 and $1500 \mathrm{nl} / \mathrm{min}$, respectively, we consider them as the single molecule traces. The threshold levels were determined by our eyes; however, the levels roughly correspond to the signal intensity in the donor channel having $\mathrm{S} / \mathrm{N}$ ratio of $\sim 5$. The traces selected after the second level of screening were considered as single molecule time series.

The intensity fluctuation of the time series obtained reflects the dynamics of sample as well as the other intensity modulations such as the non-uniformity of laser excitation and the aberration of the objective. To cancel the intensity modulations not caused by the protein dynamics, the apparent FRET efficiency time series, $E(t)$, was calculated from the fluorescence intensity time series obtained from the images for donor and acceptor channels, $I_{\mathrm{D}}(t)$ and $I_{\mathrm{A}}(t)$, respectively, based on equation (1):

$$
E(t)=\frac{I_{\mathrm{A}}(t)}{I_{\mathrm{A}}(t)+I_{\mathrm{D}}(t)}
$$

Photon count rates and noise levels in the single molecule time series. The photon count rate at one pixel of the camera, $n$, was determined by equation (2):

$$
n=\frac{C}{M} \cdot \frac{I}{t}
$$

where $I$ is the background-subtracted fluorescence intensity at one pixel, $t$ is the estimated residence time of a molecule at one pixel, $M$ is the total gain of camera, $C$ is the pre-amplifier gain on $\mathrm{A} / \mathrm{D}$ conversion. In the case for the current detector, $M$ and $C$ were 120 and 7.32 electrons/count, respectively. We ignored the dark current noise, read-out noise and background light noise, which were significantly smaller than the signal shot noise. The noise width, $\sigma$, in the time series of the apparent FRET efficiency time series, $E(t)$, was determined by the fluorescence photon counts and detector noise as described by equation (3):

$$
\sigma=F \sqrt{\frac{(1-\bar{E}) \bar{E}}{C I / M}}
$$

where $F$ is the amplification noise factor of camera. In the case for the current detector, $F$ was 1.46. $\bar{I}$ and $\bar{E}$ are the average fluorescence intensity and average apparent FRET efficiency of time series, and are defined by equations (4) and (5), respectively:

$$
\begin{gathered}
\bar{I}=\frac{1}{L} \sum_{i}^{L}\left(I_{\mathrm{A}}\left(t_{i}\right)+I_{\mathrm{D}}\left(t_{i}\right)\right), \\
\bar{E}=\frac{1}{L} \sum_{i}^{L} E\left(t_{i}\right),
\end{gathered}
$$

where $L$ is the number of points for each time series. The equation (3) was the modified form of the original expression by Dahan et al. to meet the current experimental data ${ }^{16}$. The ordinate of the relative-fluctuation plot presented in Fig. 2b and $3 \mathrm{c}$ were obtained by estimating the standard deviation of the FRET efficiency for each trace, and by dividing the value by the noise width defined by the equation (3). The abscissa of the plot was the average FRET efficiency for each trace, $\bar{E}$, defined by the equation (5).

1. Moerner, W. E. New directions in single-molecule imaging and analysis. Proc. Natl. Acad. Sci. USA 104, 12596-12602 (2007).

2. Michalet, X., Weiss, S. \& Jäger, M. Single-molecule fluorescence studies of protein folding and conformational dynamics. Chem Rev. 106, 1785-1813 (2006).

3. Roy, R., Hohng, S. \& Ha, T. A practical guide to single-molecule FRET. Nature Methods 5, 507-516 (2008).

4. Schuler, B., Lipman, E. A. \& Eaton, W. A. Probing the free-energy surface for protein folding with single-molecule fluorescence spectroscopy. Nature $\mathbf{4 1 9}$, 743-747 (2002)

5. Rhoades, E., Gussakovsky, E. \& Haran, G. Watching proteins fold one molecule at a time. Proc. Natl. Acad. Sci. USA 100, 3197-3202 (2003).

6. Kinoshita, M. et al. Development of a technique for the investigation of folding dynamics of single proteins for extended time periods. Proc. Natl. Acad. Sci. USA 104, 10453-10458 (2007).

7. Kamagata, K. et al. Long-term observation of fluorescence of free single molecules to explore protein-folding energy landscapes. J. Am. Chem. Soc. 134, 11525-11532 (2012).

8. Campos, L. A. et al. A photoprotection strategy for microsecond-resolution single-molecule fluorescence spectroscopy. Nature Methods 8, 143-146 (2011).

9. Horrocks, M. H. et al. Single molecule fluorescence under conditions of fast flow. Anal. Chem. 84, 179-185 (2012). 
10. Sato, S., Religa, T. L., Daggett, V. \& Fersht, A. R. Testing protein-folding simulations by experiment: B domain of protein A. Proc. Natl. Acad. Sci. USA 101, 6952-6956 (2004)

11. Huang, F., Sato, S., Sharpe, T. D., Ying, L. \& Fersht, A. R. Distinguishing between cooperative and unimodal downhill protein folding. Proc. Natl. Acad. Sci. USA 104, 123-127 (2007).

12. Huang, F. et al. Time-resolved fluorescence resonance energy transfer study shows a compact denatured state of the B domain of protein A. Biochemistry 48 , 3468-3476 (2009).

13. Nettels, D., Gopich, I. V., Hoffmann, A. \& Schuler, B. Ultrafast dynamics of protein collapse from single-molecule photon statistics. Proc. Natl. Acad. Sci. USA 104, 2655-2660 (2007)

14. Shaw, D. E. et al. Atomic-level characterization of the structural dynamics of proteins. Science 330, 341-346 (2010).

15. Iwakura, M., Jones, B. E., Luo, J. \& Matthews, C. R. A strategy for testing the suitability of cysteine replacements in dihydrofolate reductase from Escherichia coli. J. Biochem. 117, 480-488 (1995).

16. Dahan, M. et al. Ratiometric measurement and identification of single diffusing molecules. Chem. Phys. 247, 85-106 (1999).

\section{Acknowledgments}

S.T., K.K. and M.A. were supported in part by Grants-in-Aids for Scientific Research from MEXT. S.T. and K.K. were supported in part by the Japan Society for the Promotion of

Science (JSPS) through the "Funding Program for World-Leading Innovative R\&D on
Science and Technology (FIRST Program)" initiated by the Council for Science and Technology Policy (CSTP). M.A. was supported by Kurata Grant from the Kurata Memorial Hitachi Science and Technology Foundation, by Grant for Basic Science Research Projects from the Sumitomo Foundation, and by Japan Science and Technology Agency, PRESTO.

\section{Author contributions}

H.O., K.K., M.A. and S.T. designed the experiments. H.O. and M.S. performed the experiments. M.A. expressed and purified BdpA. H.O. and Y.S. labeled BdpA. H.O., K.K., M.A. and S.T. wrote the paper and supporting text. All authors reviewed the manuscript.

\section{Additional information}

Supplementary information accompanies this paper at http://www.nature.com/ scientificreports

Competing financial interests: The authors declare no competing financial interests.

How to cite this article: Oikawa, H. et al. Microsecond dynamics of an unfolded protein by a line confocal tracking of single molecule fluorescence. Sci. Rep. 3, 2151; DOI:10.1038/ srep02151 (2013).

(c) (i) (2) This work is licensed under a Creative Commons Attribution-

BY NG SA NonCommercial-ShareAlike 3.0 Unported license. To view a copy of this license, visit http://creativecommons.org/licenses/by-nc-sa/3.0 